\title{
User-Centric Adaptation of Multi-tenant Services: Preference-Based Analysis for Service Reconfiguration
}

\author{
Jesús García-Galán ${ }^{1}$, Liliana Pasquale ${ }^{2}$, Pablo Trinidad ${ }^{1}$, Antonio Ruiz-Cortés ${ }^{1}$ \\ ${ }^{1}$ University of Seville, Seville, Spain \\ ${ }^{2}$ Lero - the Irish Software Engineering Research Centre, University of Limerick, Ireland
}

\begin{abstract}
Multi-tenancy is a key pillar of cloud services. It allows different tenants to share computing resources transparently and, at the same time, guarantees substantial cost savings for the providers. However, from a user perspective, one of the major drawbacks of multi-tenancy is lack of configurability. Depending on the isolation degree, the same service instance and even the same service configuration may be shared among multiple tenants (i.e. shared multi-tenant service). Moreover tenants usually have different - and in most of the cases - conflicting configuration preferences. To overcome this limitation, this paper introduces a novel approach to support user-centric adaptation in shared multi-tenant services. The adaptation objective aims to maximise tenants' satisfaction, even when tenants and their preferences change during the service life-time. This paper describes how to engineer the activities of the MAPE loop to support user-centric adaptation, and focuses on the analysis of tenants' preferences. In particular, we use a game theoretic analysis to identify a service configuration that maximises tenants' preferences satisfaction. We illustrate and motivate our approach by utilising a multi-tenant desktop scenario. Obtained experimental results demonstrate the feasibility of the proposed analysis.
\end{abstract}

\section{Categories and Subject Descriptors}

H.1.2 [Information Systems Applications]: User/Machine Systems - Human information processing; H.4.2 [Information Systems Applications]: Types of Systems-Decision support

\section{General Terms}

Human Factors, Measurement

\section{Keywords}

Adaptive systems, multi-tenancy, cloud, game theory

Permission to make digital or hard copies of all or part of this work for personal or classroom use is granted without fee provided that copies are not made or distributed for profit or commercial advantage and that copies bear this notice and the full citation on the first page. To copy otherwise, to republish, to post on servers or to redistribute to lists, requires prior specific permission and/or a fee.

SEAMS '14, June 2 - 3, 2014, Hyderabad, India

Copyright 14 ACM 978-1-4503-2864-7/14/06 ...\$15.00.

\section{INTRODUCTION}

Multi-tenancy [26] allows cloud providers to deliver the same service to different tenants, which share physical and/or virtual resources transparently. Depending on the adopted cloud model, tenants can share resources at different levels, from hardware resources (e.g., CPU, storage) to software applications. Multi-tenancy can support different degrees of isolation. In particular, the lower the degree of isolation, the larger the resources and cost savings, but the smaller the configurability. The shared multi-tenant model offers the lowest degree of isolation, since the same service instance is shared by all the tenants. Such model guarantees great resources' savings, but neglects configuration capabilities, since even minimal configuration changes may have an impact on all the users. From the user perspective, this lack of configurability [22] is a major drawback of multi-tenancy, especially when users' preferences are not known in advance. Several approaches $[2,19,22,29,30]$ have been proposed to support dynamic configuration management in multi-tenant services. Nonetheless, these contributions consider an isolated multi-tenant model, and they focus on deploying different variants of existing service instances at runtime.

Recently, the notion of social adaptation [1] has been proposed to promote users as first class entities in the adaptation process. In particular, social adaptation considers changes in the users' collective judgement as a new adaptation driver. However, additional challenges have to be addressed to fully support adaptation for shared multi-tenant services. First, tenants often have different and conflicting preferences on the possible service configurations. Second, the adoption of a pay-as-you-go business model allows users to join and leave a cloud service dynamically, which may affect the global tenants' preferences. Finally, the underlying software and hardware infrastructure has a limited capacity, which may jeopardise the satisfaction of the tenants' preferences. Some of these challenges have been addressed by the software engineering community. In particular, requirements prioritisation [6, 18] and cloud infrastructure management techniques [21] provide solutions to tradeoff among conflicting stakeholders' preferences. However, to our knowledge, none of the existing approaches provides a framework to support user-centric adaptation in order to maximise the satisfaction of tenants' preferences at runtime.

Our proposal has the objective to support dynamic usercentric adaptation in shared multi-tenant services, where tenants' preferences may change at runtime and may conflict among each other. We motivate and explain the ideas presented in this paper by using a running example of a 
multi-tenant Desktop as a Service (DaaS). Since we assume a shared multi-tenant model, our approach is aimed to balance the tradeoff between tenants' satisfaction and adaptation transparency. On the one hand, the adaptation should maximise the satisfaction of the tenants' preferences, which are expressed on the configurable elements of the system. On the other hand, the adaptation should not be too intrusive i.e., the adaptation actions should have a minimal impact on the usability of the system.

The contribution of this paper is twofold. First, we define the user-centric adaptation problem in terms of challenges and activities of the MAPE (Monitoring, Analysis, Planning, Execution) loop necessary to support it. Second, we propose a preference-based analysis for identifying service configurations that maximise the tenants' satisfaction at runtime. We express the configuration space in terms of an Extended Feature Model (EFM) and the tenants' preferences by means of a preferences model [13]. The analysis is interpreted as a coalitional problem of game theory [25]. We have implemented a prototype to perform the proposed preferences analysis by leveraging metaheuristics for multiobjective optimisation [20], which have been proved suitable for EFM optimisation in existing work [16, 28, 36]. Obtained experimental results demonstrate that our preference-based analysis effectively maximises tenants' preferences and can be performed in a short time.

The rest of the paper is organised as follows. Section 2 describes the motivating scenario of a shared multi-tenant service. Section 3 illustrates the general user-centric adaptation problem, and the specific preferences-based analysis. Section 4 describes the proposed solution and Section 5 discusses experimental results. Section 6 compares our approach with relevant related work and Section 7 concludes.

\section{MOTIVATING SCENARIO}

Multi-tenancy is defined as "multiple users or processes (tenants) sharing common physical or virtual computing resources while remaining logically independent" [26]. Multitenancy is considered an essential characteristic of cloud computing, as it allows service providers to support elastic resource provisioning and pay-as-you-go billing models. However, such benefits often collide with security and configurability concerns. The more the shared resources, the higher the security concerns and the lower the configurability. On the one hand, limited configurability may have an impact on the users' satisfaction, but, on the other hand, granting users unlimited configurability options - on a service which is shared by different tenants - may even have a worse impact on users' satisfaction. For this reason, providers support different degrees of multi-tenancy, depending on factors like the users' preferences, available infrastructure, and the characteristics of the delivered service.

In this paper we have chosen the specific case of a Desktop as a Service (DaaS) as a motivating scenario. DaaS provides a virtual desktop and a set of applications-as-aservice to a single or multiple tenants. Providers like Citrix $^{1}$, VMWare ${ }^{2}$, and Amazon ${ }^{3}$ are increasingly offering a wide range of DaaS solutions. Common DaaS delivery models include Virtual Desktop Infrastructure (VDI) and hosted

\footnotetext{
${ }^{1}$ http://www.citrix.com/solutions/desktop-as-a-service/

${ }^{2}$ http://www.vmware.com/products/desktop-virtualization

${ }^{3}$ http://aws.amazon.com/workspaces/
}

shared model. The VDI model delivers a different desktop instance to each tenant, while the hosted shared model provides a different session of the same desktop instance to each tenant. These two models present benefits and drawbacks. VDI provides a more configurable DaaS with higher costs, while, despite the hosted shared model is cheaper, it has the limitation of not being flexible enough, since some tenants' preferences are intentionally neglected to avoid potentially negative side-effects on the satisfaction of the other tenants.

Table 1: Tenants' preferences (conflicting preferences are indicated by using the same number).

\begin{tabular}{|c|c|}
\hline & Preferences \\
\hline Tenant 1 & $\begin{array}{l}\text { - Aero preferred over Classic (1) } \\
\text { - Frequent Latex and Office updates } \\
\text { - Frequent antivirus checks (2) } \\
\text { - Highest firewall level (3) }\end{array}$ \\
\hline Tenant 2 & $\begin{array}{l}\text { - Classic preferred over Aero (1) } \\
\text { - Indexing and defragmentation } \\
\text { - Medium firewall level (3) } \\
\text { - No OS updates }\end{array}$ \\
\hline Tenant 3 & $\begin{array}{l}\text { - Medium frequency for office updates } \\
\text { - Frequent backups (4) } \\
\text { - Unfrequent antivirus checks (2) } \\
\text { - Medium firewall level (3) }\end{array}$ \\
\hline Tenant 4 & $\begin{array}{l}\text { - Aero preferred over Classic (1) } \\
\text { - Frequent office updates } \\
\text { - Slightly frequent backups (4) } \\
\text { - Very frequent anvirus checks (2) } \\
\text { - Highest firewall level (3) }\end{array}$ \\
\hline
\end{tabular}

As a concrete example scenario, we consider a corporate organisation that uses a hosted shared DaaS. For this scenario we have four different tenants representing the preferences of their corresponding users. Each tenant exhibits a different set of preferences (Table 1). For example, while tenants 2 and 3 prefer a medium firewall level, tenants 1 and 4 prefer the highest firewall level (conflict 1). Similarly, tenant 1 prefers Aero over Classic look \& feel, while tenant 2 has a completely opposite preference. The first challenge of this scenario is to maximise the satisfaction of the tenants' preferences. Note that the complete satisfaction of all tenants' preferences is infeasible in most of the cases, and therefore it is necessary to tradeoff between them.

Similarly to other cloud service models, DaaS satisfies the requests of its tenants elastically. This means that new users and tenants can join the system or existing ones can leave dynamically. For example, users who belong to tenant 3 work at fixed times, while the rest of the tenants accesses the DaaS at different times (including weekends), especially when project deadlines are close. Similarly, the current DaaS configuration may become sub-optimal because tenants' preferences vary during the system life-time. For example, some users of tenant 2 may prefer a lower firewall and antivirus level to run some tests. In all these cases, the current users of the DaaS and their preferences must have a direct impact on the selection of a specific service configuration. Therefore, the second challenge of this scenario is to be able to adapt the DaaS and - more in general - multi-tenant services dynamically, when user-related changes take place. 


\section{PROBLEM}

In this section, we provide a big picture of the user-centric adaptation problem (Subsection 3.1). Then, we describe the preference-based analysis, which is the specific challenge addressed in this paper (Subsection 3.2).

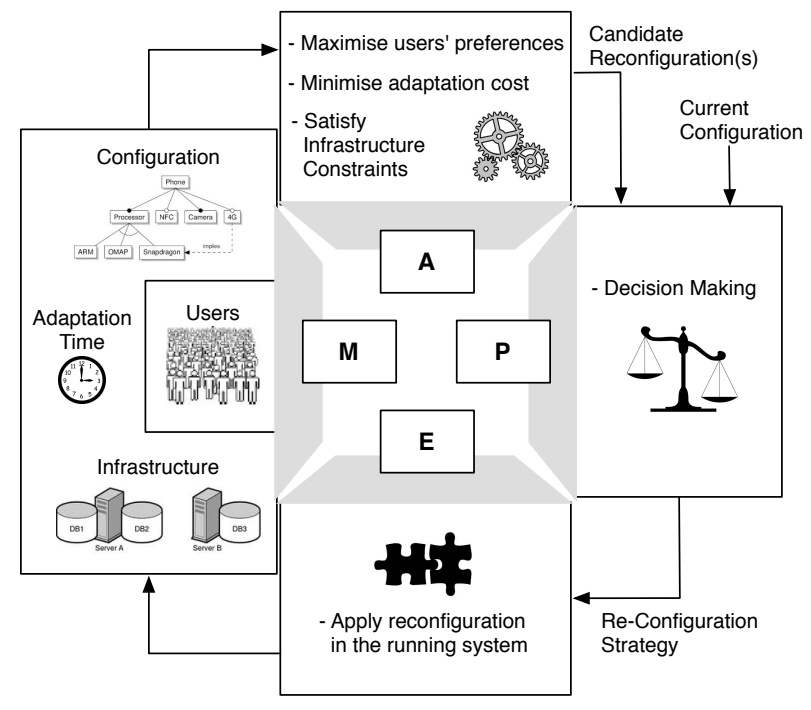

Figure 1: Used-Centric Adaptation Loop.

\subsection{Engineering User-centric Adaptation}

We define user-centric adaptation as the adaptation process aimed to continue to maximise users' satisfaction at runtime, even when the operational environment changes. These changes can affect users' preferences, available system configurations, and computational resources. As previously proposed by Ali et al. [1], users are considered as first class entities during runtime adaptation. In this paper adaptation is performed when any event that may have an impact on the users' satisfaction is detected, such as new/existing users joinining/leaving the system or modifications of users' preferences on specific system configurations. For the specific scenario proposed in this paper, user-centric adaptation aims to balance the tradeoff between satisfaction of tenants' preferences on the available system configurations and the invasiveness of the adaptation actions, which can reduce the usability of the system. Furthermore, adaptation actions perform a system re-configuration. The applicability of usercentric adaptation goes further than the hosted shared DaaS of Section 2. Even if a multi-tenant service instance is not shared by different users (e.g. a VDI DaaS), the underlying infrastructure is still shared. Therefore, user preferences on the service may have an impact in such infrastructural resources, leading to a similar scenario. Figure 1 depicts the activities of the MAPE loop necessary to support usercentric adaptation.

a) Monitoring has the objective to capture changes in the operational environment. As shown in Figure 2, these can include user-related changes, modifications of available system configurations, and variations of computational resources that can be provided by the underlining infrastructure. Any user, configuration or infrastructure related change can trigger a new adaptation. User-related changes include modifications of the users' preferences on the available system configurations or variations of the number of users per tenant, which in turn can affect the global preferences of a tenant. Moreover, user-related changes include changes of tenants' preferences and variations of the tenants that are currently using the system. Monitoring users' preferences can be performed, for example, by asking for explicit users' feedback [1] or mining the quality feedback from the users' behaviour.

Additionally, modifications of the configuration space may be due to, for example, new applications supported by the DaaS or system updates. Infrastructure changes are related to modifications of allocated resources or changes of the constraints on the maximum resources that can be allocated. Note that configuration and infrastructure changes can have an impact on how the users' preferences can be satisfied. However, since adaptation can affect the system configuration provided to each tenant, continuous adaptation can negatively affect the usability of the system [11]. For this reason, a new adaptation should only be applied if the previous one was not "too recent". To achieve this aim, the monitoring activity must also track the last time instant when an adaptation was performed.

b) Analysis has the objective to identify the best system configuration(s), which optimises a set of metrics. In particular, a candidate re-configuration should maximise the satisfaction of the users' preferences and minimise adaptation costs, which can be determined by the "intrusiveness" of a reconfiguration (i.e. how negatively a reconfiguration will affect the users' experience). For example, a re-configuration that modifies the look and feel of a DaaS instance is more intrusive than another one that modifies the number of applications that can be opened at the same time. The candidate reconfiguration(s) must also satisfy infrastructure constraints (e.g., maximum storage and computational resources that can be adopted). Analysis of users' preferences can be - and has been previously [28]- performed by using multi-objective optimisation [20] and constraints solving analysis. However, these techniques have seldom been applied at runtime.

c) Planning must compare candidate reconfiguration(s) with the current one and determine if adaptation should be performed. In case one of the candidate re-configurations is more suitable than the current one, the outcome of this activity will be an adaptation strategy, indicating how a candidate re-configuration should be applied at runtime. For example, changes in the application look and feel might not be applied until specific users terminate the interaction with the system. A re-configuration that reduces the number of applications that can be executed at the same time, for example to three, can only be applied if a user is running at most three applications at the same time.

d) Execution has the objective to apply adaptation at runtime. For example, in case of a VDI DaaS model a variant of existing application instances should be deployed dynamically, as proposed in $[2,19,22,29,30]$. While, for a hosted shared DaaS model the single application instance should be modified when possible.

\subsection{Preference-based Analysis for Service Re- configuration}

As explained previously, in this paper we focus on the analysis of tenants' preferences to reconfigure a shared multitenant service. It consists of searching for a configuration, in the service configuration space, which satisfies tenants' 


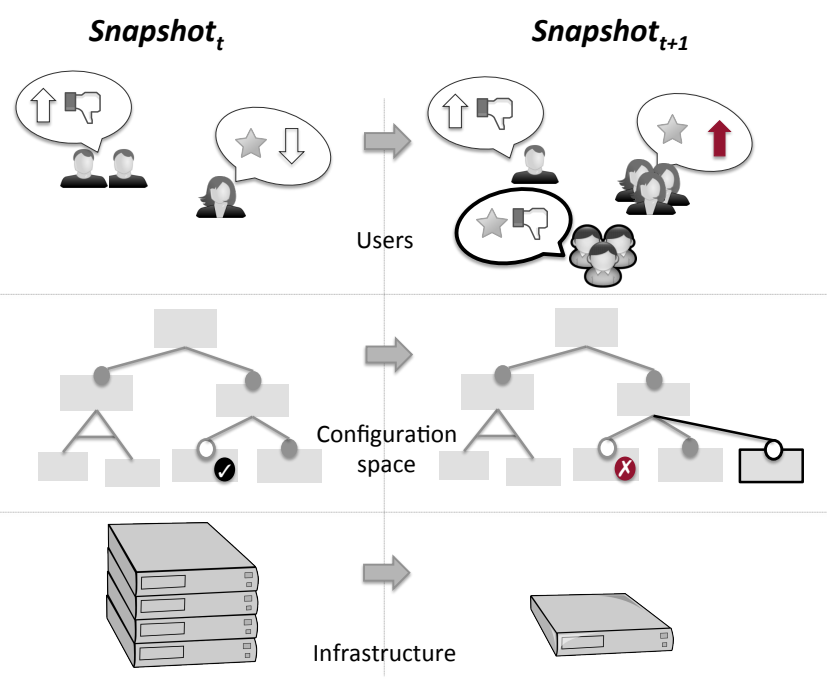

Figure 2: Changes in the operational environment captured by the monitoring activity.

preferences in a close-to-optimal way. Elasticity and multitenancy are two characteristics of cloud services that make them operate under constant changes. Tenants, their preferences, service infrastructure or even service configuration space may suffer changes in a dynamic and non-predictable way, as we described in Subsection 3.1. These changes can trigger a service reconfiguration, whose goal is to improve tenants' satisfaction.

The preferences-based analysis problem is composed of a four elements tuple

$$
A=\{C, I, U, F\}
$$

where $C$ represents the configuration space of the service, $I$ represents the underlying infrastructure, $U$ represents the utility functions of the tenants' preferences satisfaction and $F$ is a function that aggregates the utility of all the tenants for a specific configuration. $C, I$ and $U$ may change at runtime.

Given a time instant $t$ with a running configuration $c_{t-1}$, we define the analysis problem as the search of a $c_{t}$ as

$$
F\left(c_{t}\right) \geq F\left(c_{t-1}\right)+\delta
$$

where $\delta$ is a constant that measures the cost of applying the candidate reconfiguration. Tenants' preferences may change, i.e. $U_{t-1} \neq U_{t}$, and even tenants may leave or join the service. Therefore, in most of the cases the satisfaction of all the tenants may decrease $\left(F_{t}\left(c_{t-1}\right)<F_{t-1}\left(c_{t-1}\right)\right)$. As a starting point, we consider a simplified version of the analysis problem

$$
A_{1}=\{C, U, F\}
$$

where we assume that the infrastruture can support any service configuration. In other words, $C$ and $I$ are not affected by any changes, and $\delta$ is always equal to 0 . Then the only triggers of the analysis are changes in the tenants and/or their preferences.

\section{SOLUTION}

This section presents our approach for the preferencebased analysis. As shown in Figure 3, we consider potential

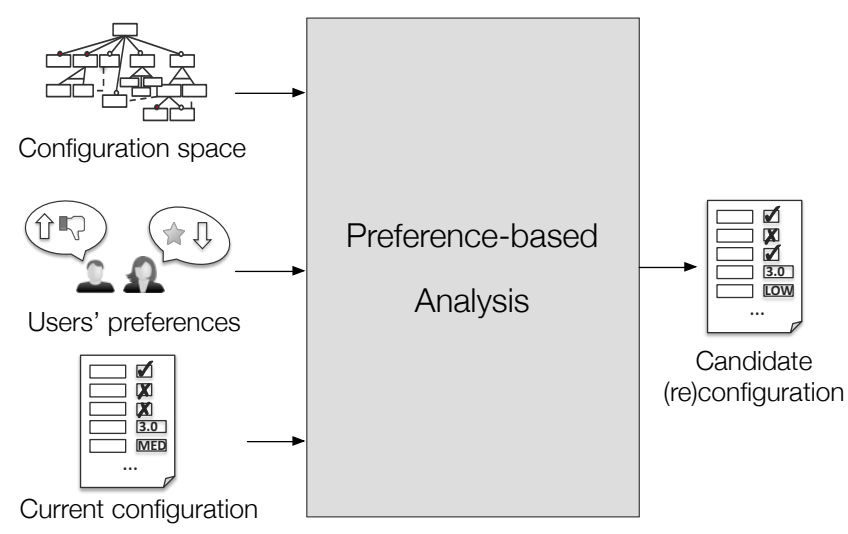

Figure 3: Inputs and outputs of the Preferencebased Analysis.

service configurations (i.e. configuration space), users' preferences expressed on the configurable service elements, and the current configuration of the service as inputs of our analysis. The configuration space is expressed as an EFM (Subsection 4.1), and the tenants' preferences are represented by using a preference model (Subsection 4.2). We interpret the analysis as a coalitional game of game theory (Subsection 4.3 ), which is solved conceptually by the Nash Bargaining Solution [25] and practically by using multi-objective optimization.

For this paper we made the assumption that every tenant groups different users who share common preferences. The clustering of the users' preferences into different tenants is performed by an external process, which is out of the scope of this work. The preferences are obtained during the monitoring phase and are translated into preferences terms, described in Section 4.2.

\subsection{Configuration Space}

The configuration space of the DaaS scenario is represented as an Extended Feature Model (EFM). An EFM represents the configuration space of a software system in terms of functional features and non-functional attributes. As shown in Figure 4, available configuration options associated with our DaaS example are Look $\&$ Feel, Security, Maintenance, and Updates.

Functional features are graphically represented as nodes and are connected among them hierarchically. They can simply be selected or not. Examples of functional features are Aero and Classic for Look $\&$ Feel. Feature relationships are individual - mandatory (black circle) and optional (white circle) - or grouped. For grouped relationships, a cardinality determines how many children features can be selected. For example, Security is mandatory, while Firewall, Encryption and Antivirus are optional features. Look 85 Feel presents a grouped relationship with a $[1,1]$ cardinality (i.e. only one sub-feature among Aero and Classic can be selected). While for application updates (AppUpdate) at least one feature among eclipse, office, and java updates should be selected.

Non-functional attributes are formally represented as variables with a domain. An attribute is linked to a feature, and optionally to other attributes by means of constraints. An example of a feature attribute can be the security level of the firewall that can assume four possible values (Low, Medium, High, and Complete). The constraint shown in Figure 4 im- 


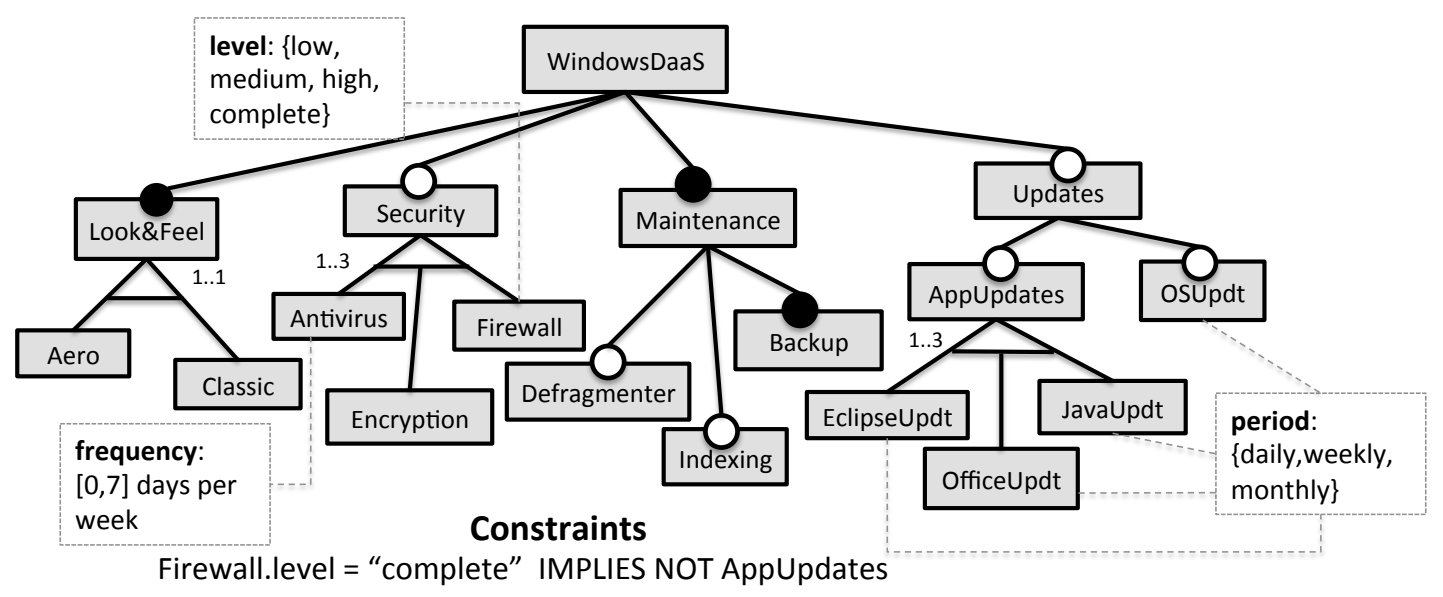

Figure 4: DaaS configuration space expressed as an Extended Feature Model

plies that, when the firewall security level is Complete, feature application updates (AppUpdates) should be disabled.

We decided to represent the configuration space as an EFM for several reasons. First, EFMs - in particular and Variability Models - in general - have been extensively used to represent variability-intensive systems for academic and industrial purposes [5]. Second, an EFM can express a large configuration space in a compact way, which is also amenable to a graphical representation. For instance, if we consider both features and attributes, the model shown in Figure 4 represents 1.053.184 different configurations ${ }^{4}$. Finally, a wide catalog of analysis operations [3] is also available to extract information from these models and, therefore, we can apply or tailor existing analysis operations to our purposes.

\subsection{Tenants' Preferences}

To express tenants' preferences we assume that the tenants agree on the hard requirements of the service. For example, in our case they agree on the operating system (Windows) and the delivery model of the DaaS (hosted shared). However, each tenant can express different preferences on the configurable service elements (i.e. features and attributes shown in Figure 4). A service cannot satisfy conflicting hard requirements, but can provide a balance between conflicting soft preferences.

We adapt some of the preference terms of Semantic Ontology of User Preferences (SOUP) preference model [12] to express the tenants' preferences. SOUP is "a highly expressive, intuitive model of user preferences". Initially, it was designed to express preferences on service discovery and rank-

\footnotetext{
${ }^{4}$ Result of Number Of Products operation [3] considering
} attributes as decision variables. ing [13]. However, it has been adapted to different scenarios, such as resources allocation in business processes [7]. SOUP defines preferences terms which refer to functional and nonfunctional terms. In our case, functional terms are features and non-functional terms are attributes. In particular, we are interested in five preferences terms:

- Favorites preference term: A favorites preference defines a finite set of values that constitute the desired values of the referred element. For our case, a favorites preferences refers to a feature we want to be selected. For example, we may favorite feature Aero.

- Dislikes preference term: A dislikes preference defines a set of property values that the service should not provide for the referred element. For our case, a dislikes preference refers to a feature we want to be removed. For example, we may dislike feature JavaUpdt.

- Highest preference term: A highest preference prefers values as high as possible for the referred element. For our case, a highest preference refers to an attribute whose value we want to maximise. For example, we may want the highest value for attribute Firewall.level.

- Lowest preference term: A lowest preference prefers values as low as possible for the referred element. For our case, a highest preference refers to an attribute whose value we want to minimise. For example, we may want the lowest value for attribute JavaUpdt.period.

- Around preference term: An around preference determines which value is better by determining the distance of each value to a concrete value provided as an operand of this preference term. For our case, an

Table 2: Adapted SOUP preferences and mapping.

\begin{tabular}{|c|c|c|l|l|}
\hline Type & Preference & Element & Mapping & Example \\
\hline \hline \multirow{2}{*}{ Qualitative } & Favorites $(\mathrm{f})$ & Feature & $f=$ selected $\Longrightarrow p_{i j}=1$ & Favorites(Aero) \\
\cline { 2 - 5 } & Dislikes $(\mathrm{f})$ & Feature & $f=$ removed $\Longrightarrow p_{i j}=1$ & Dislikes $($ JavaUpdt) \\
\hline \multirow{3}{*}{ Quantitative } & Highest $($ att $)$ & Attribute & $p_{i j}=\frac{\text { value-lowerBound }}{\text { upperBound-lowerBound }}$ & Highest $($ Firewall.level) \\
\cline { 2 - 5 } & Lowest $($ att $)$ & Attribute & $p_{i j}=\frac{\text { upperBound-value }}{\text { upperBound-lowerBound }}$ & Lowest (JavaUpdt.period) \\
\cline { 2 - 5 } & Around $($ att,d $)$ & Attribute & $p_{i j}=$ inverseDistance $($ value,$d)$ & Around (Antivirus.frequency, 3$)$ \\
\hline
\end{tabular}


around preference defines which value we want an attribute to be close to. For example, we may want attribute Antivirus.frequency to be close to 3 days.

Table 2 shows the five aforementioned preference terms, together with examples and their quantification to measure tenants' satisfaction. We have adapted SOUP preferences to work with features and attributes. Initially, described preference terms were intended to define a partial ranking between a set of items. However, we have decided to compute a satisfaction value (i.e. a real number $p_{i j} \in[0,1]$ ) for each preference. This choice allows us to compute the satisfaction of each tenant in terms of a fitness function and to compare different implementations of our analysis in order to find the best one. Since features have a boolean domain, we use both Favorites and Dislikes preferences to express features selection. Highest, Lowest and Around preferences are associated with attributes, although their domain should be ordered. For Highest and Lowest preferences, the closer the value of an attribute is to the highest or the lowest target value, respectively, the closer to 1 the value of $p_{i j}$ will be. Around is the only preference that needs an extra operand, which defines a desired value. The lower the distance of the attribute value is to such desired value, the closer to 1 the value of $p_{i j}$ will be.

\subsection{Analysis}

The goal of the analysis is to obain the configuration that best fits the different tenants' preferences. Figure 3 shows the inputs of the analysis: the service adaptation space expressed as an EFM, the tenants' preferences and the current configuration of the service. The output is a reconfiguration of the service that should satisfy current tenants' preferences better than the previous configuration.

We interpret this analysis as a problem of game theory. Game theory is a discipline which deals with "the study of mathematical models of conflict and cooperation between intelligent rational decision-makers" [23]. A game is defined as a three-elements tuple, $\Gamma=(N, C, U), i \in N$ where we have different players $(N)$ who use several strategies $\left(c_{i}, \forall i \in N\right)$ to maximise their fitness function $\left(u_{i}, \forall i \in N\right)$. Games can be cooperative or non-cooperative, depending if the players can form coalitions to accomplish better results than in an independent way. We have considered a cooperative game since we can achieve the maximum overall satisfaction of tenants' preferences. Coalitions can be ruled by communication among players, or by means of an impartial arbitrator. The solution that maximises players' fitness functions is the Nash Bargaining Solution [24, 23], which is a Pareto efficient solution maximising the Nash Product $\prod u_{i}$. We consider that all the tenants make a grand coalition and that our analysis acts as the impartial arbitrator. In particular, our analysis obtains the service configuration (Nash Bargaining Solution) that best fits tenants' preferences $(U)$ over the service adaptation space $(C)$.

Since game theory is a general discipline, we use specific techniques (i.e. multi-objective optimisation techniques) to compute a solution. We translate the tenants' preferences to fitness functions using the mapping shown in Table 2. A fitness function is expressed as $u_{i}=\sum_{j} p_{i j}$. In this way, we can measure the satisfaction of a single tenant as the sum of the satisfaction of its preferences. Each fitness function represents a different objective of the multi-objective optimisation problem. For a non-trivial multi-objective op- timisation problem, it does not exist a single solution that simultaneously optimises each objective. In this case, the objective functions are said to be conflicting, and there exists a (possibly infinite) number of Pareto optimal solutions. To choose a single solution, we apply a weighted Nash Product $\prod u_{i} * w_{i}$, where $w_{i}$ defines the weight or importance of each tenant, in order to balance the satisfaction of the different tenants. We consider specifically a Normalized Nash Product to compare different solutions from the Pareto front.

$$
N N P=\prod \frac{u_{i} * w_{i} * U_{M A X}}{U_{i M A X}}
$$

This NNP is the aggregation function $F$ defined in Subsection 3.2. $U_{i M A X}$ and $U_{M A X}$ are the maximum possible vale of $u_{i}$ for a specific tenant and the value of $u_{i}$ of all the tenants, respectively. The analysis returns the solution that maximises this product.

Table 3 shows an analysis scenario based on the DaaS example of Section 2, where $w_{i}$ is given by the number of current users of tenant $i$. In the first snapshot we have 3 tenants, and the DaaS is running a configuration $c_{1}$ which provides the satisfaction $u_{i}$ of each tenant. Note that at this stage the satisfaction of tenant 4 is very low because its preferences have not yet been taken into account during the analysis. In the second snapshot we have several changes: the preferences of tenant $_{2}$ and tenant $_{3}$ vary, there is a new tenant $\left(\right.$ tenant $\left._{4}\right)$, and the number of users of every tenant also change. Consequently, the utility value of the current configuration $\left(u_{i}\left(c_{1}\right)\right)$ changes accordingly, becoming suboptimal. Indeed the preference-based analysis is re-executed and returns a new configuration, $c_{2}$, which delivers optimal utility values $u_{i}\left(c_{2}\right)$. The most remarkable improvement is for tenant 4 , whose preferences' satisfaction increases from 0.5 to 3.5. The improvement of the global satisfaction of tenants' preferences is also indicated by the NNP (from 1.12 to 5.76$)$.

\section{EVALUATION}

This section illustrates the implementation of the prototype we adopted to perform the preferences analysis. It also describes how we conducted our experiments and discusses obtained results.

\subsection{Implementation}

We implemented a prototype to perform and evaluate the proposed users' preferences analysis. We used jMetal - a java based metaheuristics framework for multi-objective optimisation problems [10]. jMetal provides a number of algorithms to solve multi-objective optimisation problems (i.e. to compute a Pareto front of the problem). Among all the algorithms that jMetal provides, we have chosen a genetic algorithm, specifically FastPGA, due to its wide use for the analysis of EFMs [16, 28]. Since we are interested in the solution that balances and maximises tenants' satisfaction, we look to maximise the NNP defined in Section 4.3. In case at least one tenant has a satisfaction value equal to 0 , NNP returns 0. If all the returned points of the Pareto front have a $N N P=0$, then we select a solution that maximises the average satisfaction of the tenants' preferences, where the satisfaction of each tenant is weighted according to its importance (weight $w$ ). 
Table 3: Preferences reconfiguration scenario. Underlined preferences highlight changes, while blank lines represent removed preferences.

\begin{tabular}{|c|c|c|c|c|c|c|c|}
\hline & \multicolumn{3}{|l|}{ Snapshot1 } & \multicolumn{4}{|l|}{ Snapshot2 } \\
\hline & Preferences & $w_{i}$ & $u_{i}$ & Preferences & $w_{i}$ & $u_{i}\left(c_{1}\right)$ & $u_{i}\left(c_{2}\right)$ \\
\hline tenant $_{1}$ & $\begin{array}{l}\text { - Favorites(Aero) } \\
\text { - Favorites(OfficeUpdt) } \\
\text { - Around(Backup.period,Weekly) } \\
\text { - Lowest(Antivirus.frequency) } \\
\text { - Around(Firewall.level,Medium) }\end{array}$ & 45 & 3 & $\begin{array}{l}\text { - Favorites(Aero) } \\
\text { - Favorites(OfficeUpdt) } \\
\text { - Around(Backup.period,Weekly) } \\
\text { - Lowest(Antivirus.frequency) } \\
\text { - Around(Firewall.level,Medium) }\end{array}$ & 49 & 3 & 2.57 \\
\hline tenant $_{2}$ & $\begin{array}{l}\text { - Lowest(Firewall.level) } \\
\text { - Likes(Indexing) } \\
\text { - Likes(Defragmenter) } \\
\text { - Dislikes(Classic) }\end{array}$ & 60 & 3.5 & $\begin{array}{l}\text { - Around(Firewall.level,Medium) } \\
\text { - Likes(Indexing) } \\
\text { - Likes(Defragmenter) }\end{array}$ & 53 & 3 & 3 \\
\hline tenant $_{3}$ & $\begin{array}{l}\text { - Around(OfficeUpdt.period,Weekly) } \\
\text { - Highest(Antivirus.frequency) } \\
\text { - Highest(Firewall.level) }\end{array}$ & 31 & 2.5 & $\begin{array}{l}\text { - Around(OfficeUpdt.period,Weekly) } \\
\text { - Highest(Antivirus.frequency) } \\
\text { - Likes(Defragmenter) } \\
\text { - Around(Backup.period,Weekly) }\end{array}$ & 40 & 4 & 3.43 \\
\hline tenant $_{4}$ & & 0 & - & $\begin{array}{l}\text { - Likes(Classic) } \\
\text { - Around(JavaUpdt.period,Monthly) } \\
\text { - Around(Antivirus.frequency,3) } \\
\text { - Highest(Firewall.level) }\end{array}$ & 23 & 0.5 & 3.5 \\
\hline & & & & Normalized Nash Product $\left(10^{8}\right)$ & & 1.12 & 5.76 \\
\hline
\end{tabular}

Tenants' preferences are expressed using the five SOUP preferences described in Section 4.2. The set of preferences of each tenant defines the fitness function - objective - of such tenant. For the adaptation space described in Section 4.1, we have used FaMa plain text notation [33]. Since this notion only supports integer attributes at the moment, we model enumerated domains of the DaaS scenario as a range. The EFM is encoded as an array of boolean (features) and integer (attributes) variables. Since metaheuristics are approximated algorithms, they may return solutions (configurations) which violate relationships of the EFM. For this reason, taking inspiration from the work of Sayyad et al. [28], we set the correctness of the solution as an additional objective. We measure the number of violated constraints propagating each candidate solution into the EFM. Our prototype also takes as input the current configuration of the service, which is seeded among the initial population. For the first execution, we seed a random valid configuration of the service. This input is intended to have a double effect: speed up the generation of valid solutions and generate some solutions that are close to the current one and may maximise the value of the evolved fitness functions.

\subsection{Experiments}

We present a preliminary experimental study to check the feasibility of our approach. In this study, we consider scenarios where tenants are joining and leaving, and their preferences and number of users are evolving between different snapshots. For every snapshot, we run an analysis to reconfigure the service. We compare the satisfaction achieved by each reconfiguration to the satisfaction value obtained for the previous analysis. The result is also compared to the satisfaction of a random service configuration.

For our experiments, we define $T$ as a set of tenants, where each one has a number of users $w_{i}$ and a set of preferences $P_{i} . w_{i}$ is defined in the integer range $\left[W_{M I N}, W_{M A X}\right]$, considering also that $\sum w_{i} \leq W_{\text {TOT } A L}$. The number of tenants $\operatorname{card}(T)$ is defined in the integer range $\left[T_{M I N}, T_{M A X}\right]$, and the number of preferences per tenant $\operatorname{card}\left(P_{i}\right)$ is defined in the integer range $\left[P_{M I N}, P_{M A X}\right]$.

Table 4: Amount of changes between two consecutive snapshots $t-1$ and $t$.

\begin{tabular}{|r|c|c|}
\cline { 2 - 3 } \multicolumn{1}{c|}{} & $\mathbf{t}-\mathbf{1}$ & $\mathbf{t}$ \\
\hline $\operatorname{card}(T)$ & $\mathrm{n}$ & $\in\{n-1, n+1\}$ \\
$\operatorname{card}\left(P_{i}\right)$ & $m_{i}$ & $\in\left\{m_{i}-1, m_{i}, m_{i}+1\right\}$ \\
$w_{i}$ & $w_{i}$ & $\in\left[W_{M I N}, W_{M A X}\right]$ \\
\hline
\end{tabular}

To run our experiments we implemented a generator that randomly identifies a number of tenants and their preferences. Given an EFM and an integer $k$, this generator creates a set of different $k$ preferences over features and attributes of the EFM. Once a preference has been defined on an element, such element is excluded for future preferences of the same tenant to avoid contradictions. We also implemented a change scenarios generator that takes as input the set of current tenants, and returns a new set of tenants by adding/removing new/existing ones. The change scenarios generator also associates with each tenant an evolved set of preferences. We define a snapshot as the state of the tenants and their preferences for a specific time instant $t$.

Table 4 shows the amount of changes between two consecutive snapshots. For each snapshot either one tenant leaves or a new tenant joins the service, but the rest of the tenants may experience changes in their preferences. In particular, if an existing tenant is affected by a change, it can be associated with a new preference or an old preference is removed. The weight of every tenant may vary between the maximum and minimum values. We also performed experiments by using different configuration spaces. To achieve this aim, we generated four additional EFMs by using BeTTy [31], a well-known EFM generator. Table 5 shows the characteristics of the considered EFMs. CTC means the number of Cross Tree Constraints (non-hierarchical constraints) of each model.

For each EFM model shown in Table 5, we executed 25 different tenant changes scenarios. We randomised the num- 
Table 5: Characteristics of the EFMs used for our experiments.

\begin{tabular}{|r|c|c|c|c|}
\cline { 2 - 5 } \multicolumn{1}{c|}{} & Configs & Feats. & Atts. & CTC \\
BaAS & 1053184 & 18 & 7 & 1 \\
BeTTy1 & 49483952 & 20 & 12 & 6 \\
BeTT2 & $1.01 * 10^{9}$ & 20 & 14 & 6 \\
BeTTy4 & $>2.15 * 10^{9}$ & 30 & 21 & 9 \\
\hline
\end{tabular}

ber of snapshots per scenario $n$ in the integer range $[5,10]$. Initial values and ranges for the remaining parameters are as follows: $T_{\min }=2, T_{\max }=5, P_{\min }=2, P_{\max }=10$, $W_{M I N}=10$ and $W_{M A X}=80$. Since each different tenant implies a new objective, we select the same upper limit $\left(T_{\max }=5\right)$ chosen in related papers on multi-objective optimisation for Feature Models (FMs) [28, 27]. We consider $W_{\text {TOTAL }}=200$, since such value is close to the maximum number of users supported by a single real hosted shared $\mathrm{DaaS}^{5}$. For the FastPGA algorithm we configured the parameters provided by $\mathrm{j}$ Metal as follows: Evaluations $=$ 25000, PopulationSize $=100$, CrossoverProbability $=0.9$, and MutationProbability $=0.05$.

\subsection{Results and Discussion}

Table 6 shows the average execution time and average satisfaction of tenants' preferences obtained for our experiments. Execution time increases in a linear way, since we are using the same number of FastPGA evaluations for all the EFMs. However, the larger the EFM, the lower the average satisfaction achieved, because the number of evaluations remains constant. This highlights that we need to adapt the number of evaluations depending on the size of the model. We also noticed that the number of tenants does not produce any significant impact on the average execution time and tenants' satisfaction.

Table 6: Results of the preference-based analysis experimental study

\begin{tabular}{|r|c|c|c|c|}
\cline { 2 - 5 } \multicolumn{1}{c|}{} & Time (ms) & Satis. & $C_{t}>C_{R}$ & $C_{t}>C_{t-1}$ \\
\hline DaaS & 8497 & $72.79 \%$ & $98.88 \%$ & $63.48 \%$ \\
BeTTy1 & 12225 & $64.12 \%$ & $88.33 \%$ & $53.33 \%$ \\
BeTTy2 & 13701 & $59.76 \%$ & $66.67 \%$ & $38.33 \%$ \\
BeTTy4 & 18721 & $59.99 \%$ & $83.89 \%$ & $48.89 \%$ \\
\hline
\end{tabular}

$C_{t}>C_{R}$ measures the percentage of executions identifying a configuration whose satisfaction is better than a valid configuration randomly generated $\left(C_{R}\right)$. For our DaaS example, this measure reaches $98.88 \%$. Although it decreases for the rest of the models, we estimate that the reason of such decrease is also the constant number of evaluations. Figure 5 shows the average value of such measure, going from $20 \%$ to $40 \%$ depending on the model.

Finally, $C_{t}>C_{t-1}$ measures the percentage of executions that finds a reconfiguration that improves the tenants' preferences satisfaction compared to that obtained with the current configuration. We can see how this measure generally decreases for larger models. However, such results are af-

\footnotetext{
${ }^{5}$ http://blogs.citrix.com/2013/10/29/extreme-density-
} 5768-hosted-shared-desktops-in-a-single-blade-chassis/

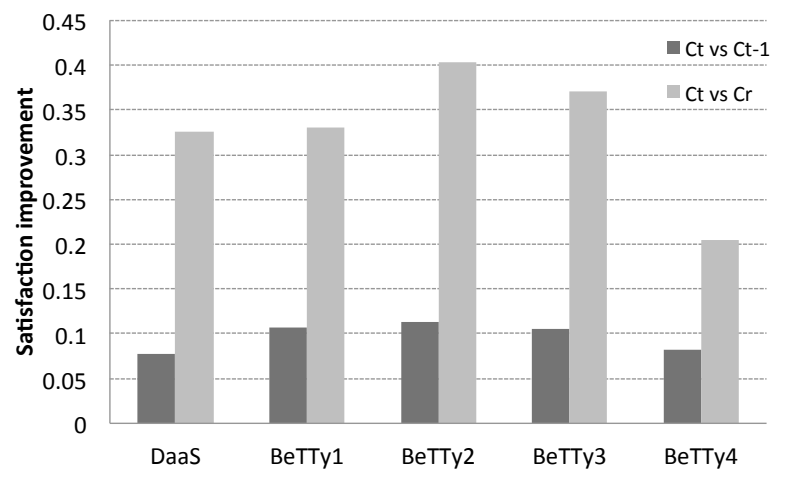

Figure 5: Comparison of the improvements achieved by our prototype between the different EFMs

fected by the "amount of change", i.e. the distance in terms of tenants and preferences between two consecutive snapshots. The larger the amount of change, the larger the subset of possible better reconfigurations. Therefore for a larger amount of changes we expect an improvement of the satisfaction of the tenants' preferences between two consecutive snapshots. However, since our prototype is only able to handle a fixed number of evaluations the average improvements in the satisfaction of the tenants' preferences between two consecutive snapshots is only around $7-10 \%$.

This is a first experimental study. In order to arrive to stronger conclusions, we need to extend this study in several ways:

1. Extend our current generator of changes scenarios to explicitly measure the amount of changes produced.

2. Try a different criteria to terminate the analysis. For example we can adapt the maximum number of evaluations depending on the size of the EFM, or consider a time limit instead.

3. Compare different jMetal algorithms and parameters. Currently we tried with FastPGA algorithm. However, depending on the amount of change or the model size, different algorithms or parameters may work better.

\section{RELATED WORK}

Users' preferences have been recently considered as a main adaptation trigger. In particular, social adaptation [1] proposes to dynamically adapt existing software systems depending on the users' collective judgement on the way the system should behave. This approach treats users' feedback as a primary driver for planning and guiding adaptation. However, they consider different kinds of feedback, while we focus on the users' preferences. The scope of the decision making is also different. Our analysis searchs a specific configuration among the whole system configuration space, while their analysis decides between the existing alternatives in order to fulfill a specific requirement. Song et al. [32] present an approach to develop self-adaptive systems that take into account end-users, who express their preferences redressing the adaptation result. In our approach, we consider an explicit preference model. Dalpiaz et al. [9] focus on user preferences over non-functional properties as a key driver for adaptation. However, this approach has its main 
focus on pervasive infrastructures and describes preferences through user routines.

Cloud services analysis and adaptation has been a prolific research area during the last years. Caton and Rana [8] propose an approach for cloud infrastructure provisioning through volunteered resources. It relies on autonomic fault management techniques to adapt resource usage. In this direction, Maurer et al. [21] also propose an adaptive resource configuration for cloud infrastructure management. In this case, they structure adaptation actions into levels, rely on Case-Based Reasoning and a rule-based approach, and even consider SLA-violations. Wei et al. [34] present a similar idea, with the difference that they use a game theoretic method based on Nash equilibria. They intend to reach an equilibrium in the differente resources allocations. Inzinger et al. [17] focus on cloud applications instead of the infrastructure. They propose a model-based adaptation which allows users to specify application behavior, and providers to consider data from multiple customers to offer better adaptation decisions.

Research on Software Product Lines (SPLs) is highly related to our paper. The idea of using variability techniques to model the adaptation space is not new. For example, Bencomo et al. [4] propose the use of variability modelling to define the runtime adaptation space. About multi-tenancy and SPLs, Schroeter et al. [29, 30] use variability and SPLs techniques to assist the configuration of multi-tenant applications. The authors identify configuration requirements and propose a configuration process using EFMs [30], and also define requirements and middleware for a variable multitenant architecture [29]. While this work focuses on the architectural aspects, our approach engineers MAPE loop and proposes a preferences-based analysis. Mietzner et al. [22] propose to use variability modelling techniques to manage the variability of Software as a Service (SaaS) applications and their requirements. Specifically, they use variability models to configure and deploy SaaS applications for different tenants. However, they focus on modelling the variability and deploying different variants of a SaaS application instance. Variability of different cloud providers has also been analysed and modelled by García-Galán et al. [14], in order to assist the migration of an in-house infrastructure to the cloud. However, this approach works with hard requirements and ignores changes of users' preferences.

Several research efforts have been made to investigate multi-objective optimisation in applications characterised by variability. Guo et al. [16] use a Genetic Algorithm to find optimal FM configurations for a single objective and user. Sayyad et al. [28, 27] perform multi-objective optimisation of several large EFMs using metaheuristics techniques. However, their objective functions are fixed (minimise errors and cost, or maximise number of features), while our fitness function depends on the specific users' preferences. Finally, other work has explored techniques for solving conflicts in a configuration process. White et al. [35] propose a technique in this direction that only considers a single user and a minimal changes criterion. While García-Galán et al. [15] consider multiple users, but after detecting the conflicts, the users have to define explicitly the impact of every solution on their preferences' satisfaction.

\section{CONCLUSIONS}

In this paper, we have presented an approach to support user-centric adaptation of multi-tenant services. We have motivated user-centric adaptation by using a DaaS example, and we have explained how to engineer the activities of the MAPE loop necessary to support it. Although our intention was to present an approach valid for any kind of adaptive system, we focused our attention on multi-tenant services and on the preference-based analysis for identifying the service configuration that maximise tenants' satisfaction. The provided solution takes inspiration from coalitional game theory. We have expressed tenants' preferences by using SOUP preference model, while the configuration space of the service is represented as an EFM. Obtained experimental results demonstrate that our analysis approach effectively maximises tenants' preferences and can be performed at runtime. However, our prototype did not exhibit good results for improving tenants' satisfaction with very large configuration spaces. This means that the time required to perform the analysis can increase with the number of configurations, and, for this reason, a different analysis technique may be required for larger configuration spaces.

As future work, firstly we propose to extend our experiments to better understand the impact of the configuration space, tenants and their evolution on the analysis results. In particular, we can compare different heuristics and algorithms to detect which one is more suitable for each specific case. Modelling more realistic multi-tenant scenarios including a larger number of configurations will also be necessary. Moreover we will extend our analysis, by including constraints in the configuration space and in the computational resources that can be provided by the infrastructure. The cost of applying a candidate reconfiguration will also be taken into account to select the best one. Finally, regarding the whole user-centric adaptation problem, we will address the rest of the activities of the MAPE loop. Indeed we will investigate how to monitor users' preferences in a non-intrusive way and how to apply a reconfiguration on the system at runtime. We plan to perform tests with users in order to determine time thresholds for specific adaptation actions.

\section{ACKNOWLEDGEMENTS}

Special thanks to J.A. Parejo for his advices for the experimental study, to J.M. Garcia for his explanations about preferences modelling, and to A. Sayyad for his useful comments about multi-objective optimisation in FMs.

This work was partially supported by the European Commission (FEDER), the Spanish and the Andalusian R\&D programmes (grants TIN2012-32273 (TAPAS), P12-TIC- 1867 (COPAS) and TIC-5906 (THEOS)), the SFI grant 10/CE/ I1855, and the ERC Advanced Grant (ASAP) no. 291652.

\section{BIBLIOGRAPHY}

[1] R. Ali, C. Solis, I. Omoronyia, M. Salehie, and B. Nuseibeh. Social Adaptation: When Software Gives Users a Voice. In Proc. of the 7th International Conference on Evaluation of Novel Approaches to Software Engineering, 2012.

[2] L. Baresi, S. Guinea, and L. Pasquale. Service-oriented dynamic software product lines. IEEE Computer, 2012. 
[3] D. Benavides, S. Segura, and A. Ruiz-Cortes. Automated analysis of feature models 20 years later: A literature review. Information Systems, 2010.

[4] N. Bencomo, P. Sawyer, G. S. Blair, and P. Grace. Dynamically adaptive systems are product lines too: Using modeldriven techniques to capture dynamic variability of adaptive systems. In Software Product Line Conference, 2008.

[5] T. Berger, A. Wasowski, K. Czarnecki, S. She, and R. Lotufo. A study of variability models and languages in the systems software domain. IEEE Transactions on Software Engineering, 2013.

[6] B. Boehm, P. Bose, E. Horowitz, and M.-J. Lee. Software requirements as negotiated win conditions. In Proceedings of the First International Conference on Requirements Engineering. IEEE, 1994.

[7] C. Cabanillas, J. M. García, M. Resinas, D. Ruiz, J. Mendling, and A. Ruiz-Cortés. Priority-based human resource allocation in business processes. In ICSOC, 2013.

[8] S. Caton and O. Rana. Towards autonomic management for cloud services based upon volunteered resources. Concurrency and Computation: Practice and Experience, 2012.

[9] F. Dalpiaz, E. Serral, P. Valderas, P. Giorgini, and V. Pelechano. A NFR-based framework for user-centered adaptation. In Conceptual Modeling. 2012.

[10] J. J. Durillo and A. J. Nebro. jMetal: A Java framework for multi-objective optimization. Advances in Engineering Software, 2011.

[11] K. Z. Gajos, M. Czerwinski, D. S. Tan, and D. S. Weld. Exploring the Design Space for Adaptive Graphical User Interfaces. In Proc. of the International Working Conference on Advanced Visual Interfaces, pages 201-208. ACM Press, 2006.

[12] J. M. García, D. Ruiz, and A. Ruiz-Cortés. An intuitive and formal description of preferences for semantic web service discovery and ranking. Technical report, University of Seville, 2012.

[13] J. M. García, M. Junghans, D. Ruiz, S. Agarwal, and A. Ruiz-Cortés. Integrating semantic web services ranking mechanisms using a common preference model. KnowledgeBased Systems, 2013.

[14] J. García-Galán, O. F. Rana, P. Trinidad, and A. RuizCortés. Migrating to the Cloud: a Software Product Line based analysis. In 3rd International Conference on Cloud Computing and Services Science (CLOSER), 2013.

[15] J. García-Galán, P. Trinidad, and A. Ruiz-Cortés. Multiuser variability configuration: A game theoretic approach. In 2013 28th IEEE/ACM International Conference on Automated Software Engineering (ASE), 2013.

[16] J. Guo, J. White, G. Wang, J. Li, and Y. Wang. A genetic algorithm for optimized feature selection with resource constraints in software product lines. Journal of Systems and Software, 2011.

[17] C. Inzinger, B. Satzger, P. Leitner, W. Hummer, and S. Dustdar. Model-based adaptation of cloud computing applications. In International Conference on Model-Driven Engineering and Software Development, 2013.

[18] J. Karlsson and K. Ryan. A cost-value approach for prioritizing requirements. Software, IEEE, 1997.

[19] I. Kumara, J. Han, A. Colman, T. Nguyen, and M. Kapuruge. Sharing with a Difference: Realizing Service-Based SaaS Applications with Runtime Sharing and Variation in Dynamic Software Product Lines. In 10th International Conference on Services Computing, 2013.
[20] R. T. Marler and J. S. Arora. Survey of multi-objective optimization methods for engineering. Structural and multidisciplinary optimization, 2004.

[21] M. Maurer, I. Brandic, and R. Sakellariou. Adaptive resource configuration for Cloud infrastructure management. Future Generation Computer Systems, 2013.

[22] R. Mietzner, A. Metzger, F. Leymann, and K. Pohl. Variability modeling to support customization and deployment of multi-tenant-aware software as a service applications. In ICSE Workshop on Principles of Engineering Service Oriented Systems, 2009.

[23] R. B. Myerson. Game theory: analysis of conflict. Harvard University Press, 1991

[24] J. Nash. Two person cooperative games. Defense Technical Information Center, 1950.

[25] J. F. Nash. The bargaining problem. Econometrica: Journal of the Econometric Society, 1950.

[26] Y. V. Natis. Gartner reference model for elasticity and multitenancy. Technical report, Gartner, Inc., 2012.

[27] A. S. Sayyad, J. Ingram, T. , Menzies, and H. Ammar. Scalable product line configuration: A straw to break the camel's back. In International Conference on Automated Software Engineering (ASE), 2013.

[28] A. S. Sayyad, T. Menzies, and H. Ammar. On the value of user preferences in search-based software engineering: a case study in software product lines. In International Conference on Software Engineering, 2013.

[29] J. Schroeter, S. Cech, S. Götz, C. Wilke, and U. Aßmann. Towards Modeling a Variable Architecture for Multi-tenant SaaS-Applications. In International Workshop on Variability Modelling of Software-Intensive Systems. ACM, 2012.

[30] J. Schroeter, P. Mucha, M. Muth, K. Jugel, and M. Lochau. Dynamic configuration management of cloud-based applications. In Proceedings of the 16th International Software Product Line Conference - Volume 2, 2012.

[31] S. Segura, J. Á.Galindo, D. Benavides, J. A. Parejo, and A. Ruiz-Cortés. BeTTy: Benchmarking and Testing on the Automated Analysis of Feature Models. In $\mathrm{VaMoS}, 2012$.

[32] H. Song, S. Barrett, A. Clarke, and S. Clarke. Selfadaptation with end-user preferences: Using run-time models and constraint solving. In Model-Driven Engineering Languages and Systems. 2013.

[33] P. Trinidad, D. Benavides, A. Ruiz-Cortés, S. Segura, and A. Jimenez. FAMA Framework. In 12th Software Product Lines Conference (SPLC), 2008.

[34] G. Wei, A. V. Vasilakos, Y. Zheng, and N. Xiong. A gametheoretic method of fair resource allocation for cloud computing services. The Journal of Supercomputing, 2010.

[35] J. White, D. Benavides, D. C. Schmidt, P. Trinidad, B. Dougherty, and Ruiz-Cortes. Automated diagnosis of feature model configurations. Journal of Systems and Software, 2010 .

[36] J. White, B. Dougherty, C. Thompson, and D. C. Schmidt. ScatterD : Spatial Deployment Optimization with Hybrid Heuristic / Evolutionary Algorithms. ACM Transactions on Autonomous and Adaptive Systems, 2011. 